\title{
COVID-19 Associated Pneumonia and Pleural Effusion Masquerading as Heart Failure in Rheumatic Heart Disease
}

\author{
$\underline{\text { Jahanzeb Malik }}{ }^{1}$, Nismat Javed ${ }^{2}$, Hesham Naeem ${ }^{1}$, Rana Abdul Sattar ${ }^{1}$, Umar Ikram $^{1}$ \\ ${ }^{1}$ Rawalpindi Institute of Cardiology, Rawal Road, Rawalpindi, Pakistan \\ ${ }^{2}$ Shifa College of Medicine, Shifa Tameer-e-Millat University, Islamabad, Pakistan
}

Received: $27 / 06 / 2020$

Accepted: $30 / 06 / 2020$

Published: $10 / 07 / 2020$

How to cite this article: Malik J, Javed N, Naeem H, Sattar RA, Ikram U. COVID-19 associated pneumonia and pleural effusion masquerading as heart failure in rheumatic heart disease. EJCRIM 2020;X: doi:10.12890/2020_001842.

Conflicts of Interests: The Authors declare that there are no competing interests.

This article is licensed under a Commons Attribution Non-Commercial 4.0 License

\section{ABSTRACT}

The ongoing outbreak of coronavirus disease 2019 (COVID-19) that began in Wuhan, China, became an international emergency when thousands of people were infected around the world. COVID-19 emerged in Pakistan in April 2020, precipitating a nationwide lockdown. While some countries are now recovering from the pandemic, its peak is not estimated to occur in Pakistan until August 2020. We present a case of rheumatic heart disease with fever, myalgia and an unusual radiological finding of the virus.

\section{LEARNING POINTS}

- Unusual radiological findings are being seen in COVID-19 patients.

- Pleural effusion in a mildly symptomatic patient is a rare presentation of the disease.

- Early aggressive treatment can produce dramatic improvement in COVID-19 pneumonia.

\section{KEYWORDS}

COVID-19, pleural effusion, rheumatic heart disease

\section{INTRODUCTION}

The outbreak of novel coronavirus disease, previously treated as pneumonia of unknown aetiology in China, was declared a pandemic in March 2020 ${ }^{[1]}$. It was named coronavirus disease 2019 (COVID-19) by World Health Organization (WHO) ${ }^{[2]}$. It arrived in Pakistan in April 2020 from Iran and spread throughout the country. With a variety of presentations including conjunctivitis, transverse myelitis, stroke and enteric symptoms, coronavirus also affects human lung parenchyma causing acute respiratory distress and pneumonia ${ }^{[3]}$. However, pleural effusion is rarely reported in COVID-19 patients ${ }^{[4]}$. Here we present the case of a 38-year-old COVID-19-positive man with rheumatic mitral valve disease and bilateral pleural effusion with pneumonia.

\section{CASE PRESENTATION}

A 38-year-old man, who was known to have rheumatic mitral valve disease for the past 4 years, attended the emergency department with a 2-day history of shortness of breath (New York Heart Association (NYHA) class II). He had severe mitral regurgitation and moderate mitral stenosis. The patient had previously refused mitral valve replacement. He was stable on medical treatment with no history of hospitalization in the past 6 months. 
His shortness of breath was associated with fever $\left(101^{\circ} \mathrm{C}\right)$ and dry cough. His heart rate was 96 beats/min and respiratory rate was 25 breaths/min. Oxygen saturation was $86 \%$ on room air. On auscultation of the lungs, bilateral coarse crepitation at the base and decreased air entry on the left side were noted. There was a displaced apex beat and a pansystolic murmur radiating to the axilla which was accentuated by expiration. His jugular vein was not distended and there was no pedal oedema.

ECG showed atrial fibrillation with a rapid ventricular rate. The chest $x$-ray revealed bilateral patchy infiltrates and pleural effusion with fluid in the right lung fissure and cardiomegaly. A complete blood count showed a total leukocyte count (TLC) of $26 \times 10^{9} /$, lymphocytes $0.5 \times 10^{9} / \mathrm{I}$ and platelet count $520 \times 10^{\%} / \mathrm{I}$. C-reactive protein (CRP) was $47 \mathrm{mg} / \mathrm{l}$, D-dimers were $1205 \mu \mathrm{g} / \mathrm{I}$ and INR was 1.4 . A peripheral smear did not show any schistocytes or fragmented red blood cells. A swab was sent for PCR testing for coronavirus.

A CT scan of the chest revealed bilateral pleural effusion with consolidation (Figs 1 and 2). On echocardiography, left ventricular function was fair with an ejection fraction of $45 \%$. There was severe mitral regurgitation and moderate mitral stenosis with a mitral valve area of $1.6 \mathrm{~cm}^{2}$ on planimetry.
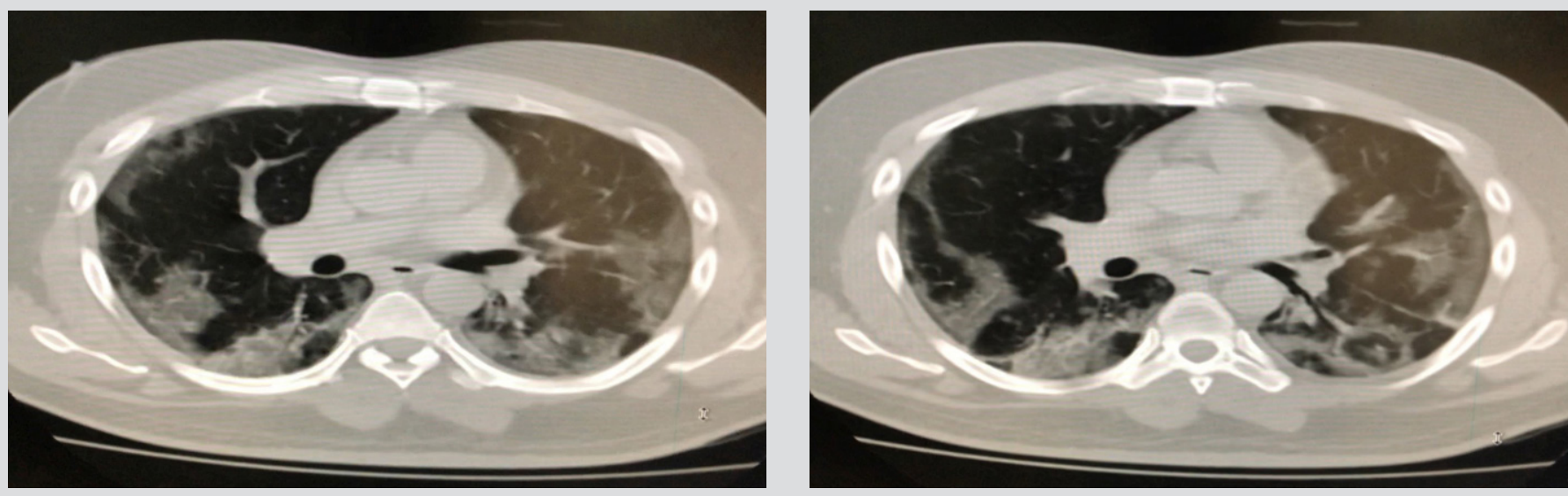

Figures 1 and 2. Consolidation and pleural effusion bilaterally

The patient was started on a furosemide infusion at $10 \mathrm{mg} / \mathrm{min}$ and azithromycin $500 \mathrm{mg}$ once daily. On the second day, he was still symptomatic, maintaining oxygen saturation on a nasal cannula at $5 \mathrm{I} / \mathrm{min}$ of oxygen. His COVID-19 PCR result was positive and he was moved to an isolation ward. After discussion with the medical specialist team, the patient was started on enoxaparin 40 mg twice daily. Actemra (tocilizumab) was given in a single dose of $400 \mathrm{mg}$. As new evidence of the efficacy of the corticosteroid dexamethasone in COVID-19 patients was emerging, he was started on $100 \mathrm{mg}$ twice daily.

On the fourth day, the patient became afebrile. His chest x-ray improved and pleural effusion subsided to only the left side. His oxygen saturation started to improve and his furosemide infusion was stopped. His CRP dropped to $9 \mathrm{mg} / \mathrm{l}$, D-dimer to $560 \mu \mathrm{g} / \mathrm{l}$, and TLC to $15 \times 10^{9} / \mathrm{l}$. On the sixth day, he was discharged on furosemide $40 \mathrm{mg}$ twice daily, Aldactone $25 \mathrm{mg}$ once daily, warfarin $2.5 \mathrm{mg}$ once daily, and prednisolone $30 \mathrm{mg}$ daily in divided doses. After cardiac stabilization and the agreement of the medical specialist at our institute, he was referred to a COVID-19-designated hospital for further management.

\section{DISCUSSION}

With the worldwide spread of COVID-19, uncommon presentations are still being reported. In our case, all clinical characteristics including signs and symptoms, laboratory investigations, and the results of CT of the chest suggested COVID-19. The diagnosis was confirmed on COVID-19 PCR. However, pleural effusion, which was diagnosed on CT of the chest, is rare in the early phase of the disease ${ }^{[5]}$. It usually occurs with recurrent pneumonia or after the third week of COVID-19 pneumonia ${ }^{[6]}$. At this stage, patients are severely sick compared with other patients. A study of chest CT scans has shown that viral pneumonia has a higher incidence of pleural effusion than COVID-19 pneumonia (39\% vs. $4 \%)^{[7]}$. A similar study reported that the percentage of pleural effusion is $8 \%$ in patients with mild symptoms compared with $28 \%$ in patients critically ill with COVID-19[8].

Our patient presented with mild symptoms at an early stage and his medical examination and CT revealed pleural effusion. Although most patients have mild symptoms and a good prognosis, COVID-19 can develop into severe illness including pneumonia, pulmonary oedema, acute respiratory distress syndrome, multiple organ failure, and even death. 


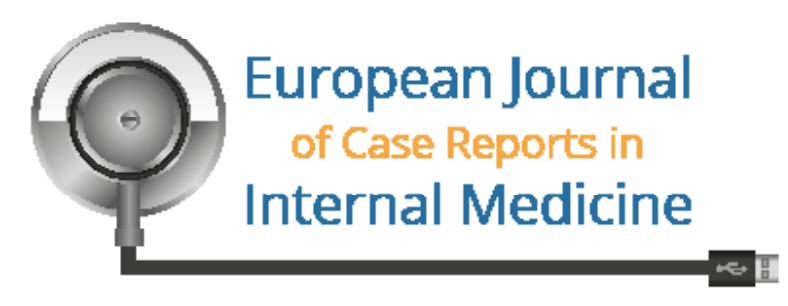

Our patient was promptly treated with newer on-trial drugs available at our institute as he was already compromised owing to his rheumatic heart disease. Proper investigations and early aggressive treatment can prevent the devastating sequelae of this disease.

\section{CONCLUSION}

The virus can have a variety of presentations with unusual radiological findings. Keen clinical judgment with appropriate investigations is mandatory for prompt diagnosis and treatment.

\section{REFERENCES}

1. Wang W, Tang J, Wei F. Updated understanding of the outbreak of 2019 novel coronavirus (2019-nCoV) in Wuhan, China. J Med Virol 2020;92:441-447.

Velavan TP, Meyer CG. The COVID-19 epidemic. Trop Med Int Health 2020;25:278-280.

3. Fried JA, Ramasubbu K, Bhatt R, Topkara VK, Clerkin KJ, Hornet E, al. The variety of cardiovascular presentations of COVID-19. Circulation 2020;141:1930-1936.

4. Li K, Wu J, Wu F, Guo D, Chen L, Fang Z, et al. The clinical and chest CT features associated with severe and critical COVID-19 pneumonia. Invest Radiol 2020;55(6):327-331.

5. Bao C, Liu X, Zhang H, Li Y, Liu J. Coronavirus disease 2019 (COVID-19) CT findings: a systematic review and meta-analysis. J Am Coll Radiol 2020;17:701-709.

6. Shi H, Han X, Jiang N, Cao Y, Alwalid O, Gu J, et al. Radiological findings from 81 patients with COVID-19 pneumonia in Wuhan, China: a descriptive study. Lancet Infect Dis 2020;20(4):425-434.

7. Lal A, Mishra AK, Sahu KK. CT chest findings in coronavirus disease-19 (COVID-19). J Formos Med Assoc 2020;119(5):1000-1001.

8. Han R, Huang L, Jiang H, Dong J, Peng H, Zhang D. Early clinical and CT manifestations of coronavirus disease 2019 (COVID-19) pneumonia. AJR Am J Roentgenol 2020;1:6. 